Article

\title{
In Vivo Protective Effects of Diosgenin against Doxorubicin-Induced Cardiotoxicity
}

\section{Chih-Tai Chen ${ }^{1, \dagger}$, Zhi-Hong Wang ${ }^{2, \dagger}$, Cheng-Chin Hsu ${ }^{1}$, Hui-Hsuan Lin ${ }^{3,4, *}$ and Jing-Hsien Chen ${ }^{1,4, *}$}

1 School of Nutrition, Chung Shan Medical University, Taichung City 40201, Taiwan; E-Mails: thomas.chen@unitybiotech.com (C.-T.C.); king@csmu.edu.tw (C.-C.H.)

2 Environment-Omics-Diseases Research Center, China Medical University Hospital, Taichung City 40402, Taiwan; E-Mail: rover_wang@hotmail.com

3 School of Medical Laboratory and Biotechnology, Chung Shan Medical University, Taichung City 40201, Taiwan

4 Clinical Laboratory, Chung Shan Medical University, Taichung City 40201, Taiwan

$\dagger$ These authors contributed equally to this work.

* Authors to whom correspondence should be addressed; E-Mails: cjh0828@csmu.edu.tw (J.-H.C.); linhh@csmu.edu.tw (H.-H.L.); Tel.: +886-4-24730022 (ext. 12195) (J.-H.C.); +886-4-24730022 (ext. 12410) (H.-H.L.); Fax: +886-4-23248175 (J.-H.C.); +886-4-23248171 (H.-H.L.)

Received: 24 April 2015 / Accepted: 8 June 2015 / Published: 17 June 2015

\begin{abstract}
Doxorubicin (DOX) induces oxidative stress leading to cardiotoxicity. Diosgenin, a steroidal saponin of Dioscorea opposita, has been reported to have antioxidant activity. Our study was aimed to find out the protective effect of diosgenin against DOX-induced cardiotoxicity in mice. DOX treatment led to a significant decrease in the ratio of heart weight to body weight, and increases in the blood pressure and the serum levels of lactate dehydrogenase (LDH), creatine phosphokinase (CPK) and creatine kinase myocardial bound (CK-MB), markers of cardiotoxicity. In the heart tissue of the DOX-treated mice, DOX reduced activities of antioxidant enzymes, including superoxide dismutase (SOD) and glutathione peroxidase (GPx), were recovered by diosgenin. Diosgenin also decreased the serum levels of cardiotoxicity markers, cardiac levels of thiobarbituric acid relative substances (TBARS) and reactive oxygen species (ROS), caspase-3 activation, and mitochondrial dysfunction, as well as the expression of nuclear factor kappa B (NF- $\kappa \mathrm{B})$, an inflammatory factor. Moreover, diosgenin had the effects of increasing the cardiac levels of
\end{abstract}


cGMP via modulation of phosphodiesterase-5 (PDE5) activity, and in improving myocardial fibrosis in the DOX-treated mice. Molecular data showed that the protective effects of diosgenin might be mediated via regulation of protein kinase A (PKA) and p38. Our data imply that diosgenin possesses antioxidant and anti-apoptotic activities, and cGMP modulation effect, which in turn protect the heart from the DOX-induced cardiotoxicity.

Keywords: doxorubicin; cadiotoxicity; diosgenin; antioxidant; cGMP

\section{Introduction}

Doxorubicin (DOX) belongs to the family of anthracyclines, and has been used to against cancer since late 1960s. It is the most effective anticancer drugs. However, studies of cardiotoxic effects of DOX have been reported [1,2]. Therefore, chemotherapy with DOX is limited by its cardiotoxicity. The development of cumulative dose-dependent cardiomyopathy may occur many years after the cessation of DOX treatment. It has been calculated that approximately $10 \%$ of patients treated with DOX will develop cardiomyopathy [3,4]. Multiple mechanisms are involved in DOX induced cardiomyopathy, such as the increase in cardiac oxidative stress and lipid peroxidation, and changes in adenylate cyclase activity leading to apoptosis and inflammation-related signaling pathway [5,6]. In previous research, free radical scavengers including probucol, vitamin $\mathrm{E}$ and ellagic acid have been demonstrated protection from DOX-induced cardiotoxicity, indicating the roles of reactive oxygen species (ROS) and nuclear factor kappa B (NF-kB) [7-9]. Acute DOX cardiotoxicity involves cardiomyocyte apoptosis. It is generally agreed that the elevated oxidative stress induced by DOX activates signaling pathway leading to cardiomyocyte apoptosis [10]. Caspase activity can be influenced by DOX, and caspase-3 activation is associated with DOX administration [11]. Thus, apoptosis plays a role in the development of heart failure via a loss of cardiomyocyte.

Due to the importance of DOX in the chemotherapy treatment for many types of cancer, strategies have been tried to prevent or attenuate the side effects of DOX administration, including the use of DOX analogues, alternative drug-delivery methods, and the iron-chelating agent. But so far, the ability of treatments to prevent or attenuate DOX-induced damage has been limited. Therefore, discovery of novel agents for reducing its side effects is still needed. Several studies in recent years suggest that sildenafil, a selective inhibitor of cyclic guanosine monophosphate (cGMP)-specific phosphodiesterase5 (PDE5), may give some help against the DOX-induced cardiomyopathy [12,13]. PDE5 inhibitors have been shown to play a critical role against cardiac ischemia-reperfusion (I/R) injuries by activating cGMP-dependent protein kinase signaling pathway [14]. In the heart, cGMP modulates vascular tone, platelet function, cardiomyocyte contraction, mitochondrial function, and stress-response signaling [15].

Diosgenin is a steroidal saponin found in yam [16], the edible tubers of Dioscorea opposita and one of the most used plants in the world. Diosgenin has been shown to have favorable effects on anti-inflammatory [17], lipid metabolism [18], glucose lowering [19], and antioxidant activities [20,21]. Previous studies indicated that a supplementation of food rich in diosgenin, such as a yam variety (called air potato), has been shown to possess the protective effect on myocardial I/R injury in rats due to apoptosis and necrosis [22]. In the literature, diosgenin effectively protected against isoproterenol-induced 
myocardial necrosis in rats [23]. Moreover, the recent study found that diosgenin abrogated production of intracellular ROS [24]. Another finding suggests that diosgenin has a beneficial role against aortic remodeling induced by oxidative stress in diabetic state and decreases the lipid peroxidation in aorta [25]. Based on the potential role of diosgenin in ameliorating oxidative stress and injury, we attempted to evaluate the beneficial effects of diosgenin against the DOX-induced cardiotoxicity in mice.

\section{Experimental Section}

\subsection{Chemicals}

Doxorubicin hydrochloride and diosgenin were purchased from Sigma-Aldrich Co. (St. Louis, MO, USA). All chemicals used in this study were purchased from the commercially available.

\subsection{Animals and Experimental Protocol}

Male Balb/c mice, 4-5 weeks old, were obtained from National Laboratory Animal Center (National Science Council, Taipei City, Taiwan). Use of the mice was approved by the guidelines of the Instituted Animal Care and Use Committee of Chung Shan Medical University (IACUC, CSMU). Mice were housed on a 12-h light/dark cycle and fed with mouse standard chow diet (MF-18, Oriental Yeast Co., Ltd. Tokyo, Japan), and then started the experiments after 1-week acclimation. The mice were randomly divided into three groups (ten mice per group) and treated as follows: Group 1, vehicle (normal control); Group 2, DOX at $3 \mathrm{mg} / \mathrm{kg}$ of body weight once a week, i.p.; Group 3, DOX with diosgenin at $130 \mathrm{mg} / \mathrm{kg}$ of body weight once daily, p.o. (DOX + diosgenin). DOX was administered intraperitoneally to the mice of Groups 2 and 3 at a dose of $3 \mathrm{mg} / \mathrm{kg}$ once a week for 4 weeks (a total of $12 \mathrm{mg} / \mathrm{kg}$ ). At the same time, Group 3 was treated with oral feeding of diosgenin at doses of $130 \mathrm{mg} / \mathrm{kg}$ daily for 4 weeks. The doses and injection regiments for these drugs were based on the reports published previously [26] with some modification. At the end of 4 weeks, mice were euthanized by carbon-dioxide asphyxiation followed by exsanguination. The hearts were excised and weighed, and serum and cardiac samples were collected and used for analysis as described below.

\subsection{Heart Rate, Blood Pressure Monitoring, and Blood Analysis}

Heart rate and blood pressure was performed by tail cuff method using Blood Pressure Monitor for rats and mice (Model MK 2000- Muromachi Kikai Co. Ltd., Tokyo, Japan). Serum lactate dehydrogenase (LDH) activity was assayed by commercial kits (Randox, Crumlin, UK). Serum levels of creatine phosphokinase (CPK) and creatine kinase myocardial bound (CK-MB) were determined according to standard methods using diagnostic kits from BioSystems S.A. (Barcelona, Spain).

\subsection{Measurement of Thiobarbituric Acid Relative Substances (TBARS), ROS and Antioxidant Status in Heart}

TBARS (nmol/mg protein) level in cardiac tissue was determined by fluorescence spectrophotometer (excitation at $532 \mathrm{~nm}$ and emission at $600 \mathrm{~nm}$ ) as described previously [27]. Quantification of TBARS was performed by comparison with a standard dosage of malondialdehyde (MDA), the lipid peroxidation 
product, which is generated by acid-catalyzed hydrolysis of 1,1,3,3-tetramethoxypropane. ROS in cardiac tissue was measured by using commercial kits (Calbiochem Inc., San Diego, CA, USA). Cardiac activities of glutathione peroxidase (GPx) and superoxide dismutase (SOD) were determined by commercial assay kits (Calbiochem Inc., San Diego, CA, USA), and glutathione (GSH) by commercial assay kits (OxisResearch, Portland, OR, USA).

\subsection{Enzyme Immunoassay}

Levels of cAMP and cGMP in the heart tissue were assayed with a competitive enzyme immunoassay (Cayman Chemical, Ann Arbor, MI, USA) according to the manufacturer's protocol.

\subsection{Real-Time Polymerase Chain Reaction (Real-Time PCR) for mRNA Expression}

Total RNA was isolated from cells with a guanidinium chloride procedure as described previously, and the mRNA levels were analyzed by real-time quantitative RT-PCR using a Bio-Rad iCycler system (Bio-Rad, Hercules, CA, USA) [28]. The mRNAs were reverse-transcribed into cDNAs by using an iScript cDNA synthesis kit (Bio-Rad). The specificity of primers was tested by running a regular PCR for 40 cycles at $95{ }^{\circ} \mathrm{C}$ for $20 \mathrm{~s}$ and $60{ }^{\circ} \mathrm{C}$ for $1 \mathrm{~min}$ followed by electrophoresis on an agarose gel. The real-time PCR was performed using a SYBR supermix kit (Bio-Rad) and run for 40 cycles at $95{ }^{\circ} \mathrm{C}$ for $20 \mathrm{~s}$ and $60^{\circ} \mathrm{C}$ for $1 \mathrm{~min}$. Each 20 - $\mu \mathrm{L}$ PCR mixture contained cDNA template, SYBR supermix kit, and $0.5 \mu \mathrm{M}$ of each gene-specific primer. Specific primers were designed using Beacon Designer 2.0 software (Table 1). The PCR efficiency was examined by serial dilution of the cDNA, and the PCR specificity was checked by melting curve data. Each cDNA sample was triplicated and the corresponding no-RT mRNA sample was included as a negative control. The GADPH primers were included in every plate to avoid sample variations. The mRNA level of each sample for each gene was normalized to that of the GADPH mRNA.

Table 1. Sequences of different primers used for real-time PCR reactions.

\begin{tabular}{|c|c|c|}
\hline Gene & Primer & $\begin{array}{c}\text { Sequence } \\
\end{array}$ \\
\hline \multirow{2}{*}{ PDE5A } & Forward & 5'-AAATGGTGGGACCTTCACTG-3' \\
\hline & Reverse & 5'-GTGGCCGCTATCTTCTTCAG-3' \\
\hline \multirow{2}{*}{ PDE3A } & Forward & 5'-AATGTGGCCGTATTCTGAGC-3' \\
\hline & Reverse & 5'-GAATCGGCTGTGTTGTGAGA-3' \\
\hline \multirow[b]{2}{*}{$\mathrm{NF}-\kappa \mathrm{B}$} & Forward & 5'-CAGACCGCAGTATCCATAGC-3' \\
\hline & Reverse & 5'-CGTGAAAGGGGTTATTGTTGG-3' \\
\hline \multirow{2}{*}{ TGF- $\beta$} & Forward & 5'-TGACGTCACTGGAGTTGTACGG-3' \\
\hline & Reverse & 5'-GGTTCATGTCATGGATGGTGC-3' \\
\hline \multirow{2}{*}{ GADPH } & Forward & 5'-TGTGTCCGTCGTGGATCTGA-3' \\
\hline & Reverse & 5'-TTGCTGTTGAAGTCGCAGGAG-3' \\
\hline
\end{tabular}

\subsection{Protein Preparation and Western Blot Analysis}

Proteins from the heart tissues were extracted in RIPA buffer (1\% Triton X-100, $150 \mathrm{mmol} / \mathrm{L} \mathrm{NaCl}$, $5 \mathrm{mmol} / \mathrm{L}$ EDTA, and $10 \mathrm{mmol} / \mathrm{L}$ Tris-HCl, $\mathrm{pH}$ 7.0) containing a protease inhibitor cocktail. Protein extracts were subjected to centrifugation at $10,000 \mathrm{~g}$ for $10 \mathrm{~min}$. Total protein (10-50 $\mu$ g per lane) was electrophoresed and separated on 8\%-15\% SDS-poly-acrylamide gels and transferred to nitrocellulose 
membranes. After blocking with 5\% nonfat dry milk, the membranes were incubated with the indicated primary antibodies overnight at $4{ }^{\circ} \mathrm{C}$. The blots were incubated with the antibodies against caspase- 3 , phospho-PKA, PKA, phospho-p38, p38 and $\beta$-actin, purchased from Santa Cruz Biotechnology, Inc. (Santa Cruz, CA, USA). $\beta$-actin served as an internal control. The blot was quantified by enhanced chemiluminescence detection (Amersham Pharmacia Biotech, Little Chalfont, Bucks, UK).

\subsection{Isolation of Mitochondria and Cytochrome C Assay}

The preparation of cytosolic and mitochondrial fractions of cardiac tissue was performed as described previously [29]. The isolated hearts were washed in sterile PBS and the mitochondria were isolated according to the manufacturer's instructions (Mitochondria Isolation Kit for Tissue; Pierce, Rockford, IL, USA). Briefly, tissues were minced after addition $800 \mathrm{~mL}$ Mitochondria Isolation Reagent A and carefully homogenized with 20 strokes on ice. The crude homogenates were then returned to the original tube, and $800 \mathrm{~mL}$ Mitochondria Isolation Reagent $\mathrm{C}$ was added. The tube was centrifuged at $700 \mathrm{~g}$ for $10 \mathrm{~min}$ at $48{ }^{\circ} \mathrm{C}$. The supernatant was transferred and centrifuged at $3000 \mathrm{~g}$ for $15 \mathrm{~min}$ at $48{ }^{\circ} \mathrm{C}$ to obtain a more purified fraction of mitochondria. The resulting supernatant was transferred into a new tube and centrifuged at 12,000 g to produce a more purified cytosolic fraction and saved for cytochrome c assay. The pellet contained the isolated mitochondria. Mitochondria Isolation Reagent C $(500 \mathrm{~mL})$ was added to the pellet, and the mixture was centrifuged at 12,000 $\mathrm{g}$ for $5 \mathrm{~min}$. The mitochondrial pellet was resuspended in $300 \mathrm{~mL}$ of mitochondria isolation buffer containing $0.1 \%$ Triton X-100 and protease inhibitors. Protein concentrations of both mitochondrial and cytosolic lysates were determined using BCA Protein Assay Reagents (Pierce, Rockford, IL, USA). To detect cytochrome c release into the cytosol, Western blotting was performed with antibodies against cytochrome c and COX IV, a mitochondrial marker (Santa Cruz, CA, USA).

\subsection{Statistical Analysis}

Results from ten mice ( $n=10)$ were analyzed and expressed as means \pm SD. Statistical analysis was done using one-way ANOVA, and post hoc comparisons were carried out using Duncan's multiplerange test. $p<0.05$ was considered statistically significant.

\section{Results}

\subsection{Diosgenin Diminished DOX-Induced Losses of Heart Weight, the Ratio of Heart Weight to Body Weight, and Heart Rate, and Attenuated DOX-Increased Blood Pressure, and Serum Levels of Cardiotoxicity Markers}

Effect of DOX on heart weight, body weight, and the ratio of heart weight to body weight are shown in Table 2. The heart weight and the ratio of heart weight to body weight were significantly decreased by DOX treatment, but diosgenin treatment diminished the DOX-induced loss in heart weight by $48.7 \%$ $(p<0.05)$. The decrease in the ratio of heart weight to body weight due to DOX treatment was also alleviated by diosgenin in the DOX plus diosgenin group comparing to the DOX group $(p<0.05)$. Heart rate and blood pressure were performed after the four weeks of DOX administration. Table 2 also shows that DOX treatment resulted in significant cardiac functional deterioration as characterized by increase 
in blood pressure and lower heart rate as compared with normal group ( $p<0.05)$. LDH, CPK, and CK-MB are important clinical markers of cardiac injury [30]. As expected, serum levels of LDH, CPK and CK-MB were significantly elevated in the DOX alone treated group as compared with the control $(p<0.05)$. Treatment with diosgenin in the DOX plus diosgenin group significantly reduced their levels as compared with the DOX alone treated group $(p<0.05)$.

Table 2. Effect of diosgenin on body weight, heart weight, ratio of heart weight to body weight, heart rate, blood pressure, and serum levels of lactate dehydrogenase (LDH), creatine phosphokinase (CPK) and creatine kinase myocardial bound (CK-MB) in doxorubicin (DOX)-treated mice.

\begin{tabular}{cccc}
\hline & Normal & DOX & DOX + Diosgenin \\
\hline Body weight (g) & $28.00 \pm 0.00^{\mathrm{b}}$ & $20.77 \pm 3.64^{\mathrm{a}}$ & $21.73 \pm 2.84^{\mathrm{a}}$ \\
Heart weight (mg) & $166.43 \pm 15.82^{\mathrm{b}}$ & $131.78 \pm 21.74^{\mathrm{a}}$ & $148.05 \pm 15.51^{\mathrm{ab}}$ \\
Heart weight/body weight ratio $^{\mathrm{b}}$ & $5.94 \pm 0.57^{\mathrm{b}}$ & $4.66 \pm 1.05^{\mathrm{a}}$ & $5.83 \pm 0.75^{\mathrm{b}}$ \\
Heart rate (bpm) ${ }^{\mathrm{ab}}$ & $611.40 \pm 15.65^{\mathrm{b}}$ & $566.31 \pm 27.36^{\mathrm{a}}$ & $595.59 \pm 32.83^{\mathrm{ab}}$ \\
Blood pressure (mmHg) & $98.08 \pm 11.40^{\mathrm{a}}$ & $119.93 \pm 26.25^{\mathrm{b}}$ & $98.88 \pm 15.49^{\mathrm{a}}$ \\
Serum LDH (U/L) & $173.02 \pm 8.70^{\mathrm{a}}$ & $263.91 \pm 54.35^{\mathrm{b}}$ & $152.13 \pm 29.25^{\mathrm{a}}$ \\
Serum CPK (U/L) & $106.31 \pm 17.06^{\mathrm{a}}$ & $241.22 \pm 18.77^{\mathrm{b}}$ & $165.32 \pm 16.02^{\mathrm{ab}}$ \\
Serum CK-MB (U/L) & $86.90 \pm 6.72^{\mathrm{a}}$ & $154.28 \pm 15.03^{\mathrm{b}}$ & $107.15 \pm 10.33^{\mathrm{ab}}$ \\
\hline
\end{tabular}

Values are mean $\pm \mathrm{SD}, n=10{ }^{\mathrm{a}, \mathrm{b}}$ Means in a row without a common letter differ, $p<0.05$. \#: beats per minute.

\subsection{Diosgenin Restored DOX-Induced Alterations in Oxidative Status}

To confirm the induction of oxidative stress by DOX, tissue lipid peroxidation, antioxidation and antioxidant enzymes were also evaluated. As shown in Table 3, DOX treatment increased TBARS and ROS levels, decreased GSH content as well as GPx and SOD activities in the heart. As compared with DOX alone, diosgenin treatment lowered ROS and MDA levels, retained GSH content, and recovered cardiac GPX and SOD activities $(p<0.05)$ (Table 3).

Table 3. Effect of diosgenin on cardiac levels of thiobarbituric acid relative substances (TBARS) and reactive oxygen species (ROS), and glutathione (GSH), and activities of glutathione peroxidase (GPx) and superoxide dismutase (SOD) in DOX-treated mice.

\begin{tabular}{cccc}
\hline & Normal & DOX & DOX + Diosgenin \\
\hline TBARS (nmol/mg protein) & $0.16 \pm 0.02^{\mathrm{a}}$ & $0.27 \pm 0.07^{\mathrm{b}}$ & $0.17 \pm 0.04^{\mathrm{a}}$ \\
ROS (RFU/mg protein) & $1.02 \pm 0.16^{\mathrm{a}}$ & $1.51 \pm 0.50^{\mathrm{b}}$ & $1.05 \pm 0.16^{\mathrm{a}}$ \\
GSH (nmol/mg protein) & $5.97 \pm 0.28^{\mathrm{b}}$ & $2.36 \pm 0.99^{\mathrm{a}}$ & $4.43 \pm 1.37^{\mathrm{ab}}$ \\
GPx (U/mg protein) & $43.79 \pm 0.73^{\mathrm{b}}$ & $27.52 \pm 3.42^{\mathrm{a}}$ & $40.19 \pm 4.75^{\mathrm{b}}$ \\
SOD (U/mg protein) & $4.29 \pm 0.64^{\mathrm{b}}$ & $2.90 \pm 0.92^{\mathrm{a}}$ & $3.94 \pm 0.51^{\mathrm{ab}}$ \\
\hline
\end{tabular}

Values are mean $\pm \mathrm{SD}, n=10$. ${ }^{\mathrm{a}, \mathrm{b}}$ Means in a row without a common letter differ, $p<0.05$. 


\subsection{Diosgenin Improved DOX-Regulated Levels of cAMP and cGMP, and mRNA Expression of PDE5A}

It is well known that cGMP exerts its effects by interacting with PDE, and cGMP-dependent signaling plays an important protective role in cardiac injury [14,15]. As compared with the control, treatment with DOX decreased the levels of cAMP and cGMP in the heart, especially the later $(p<0.05)$ (Figure 1A). Diosgenin augmented the cGMP and cAMP levels as compared with DOX alone $(p<0.05)$. Moreover, the cardiac mRNA expression of PDE5A was increased after a four-week treatment of DOX, whereas that of PDE3A was slightly affected. Treatment with diosgenin also attenuated both mRNA expressions compared to DOX alone $(p<0.05)$ (Figure 1B).

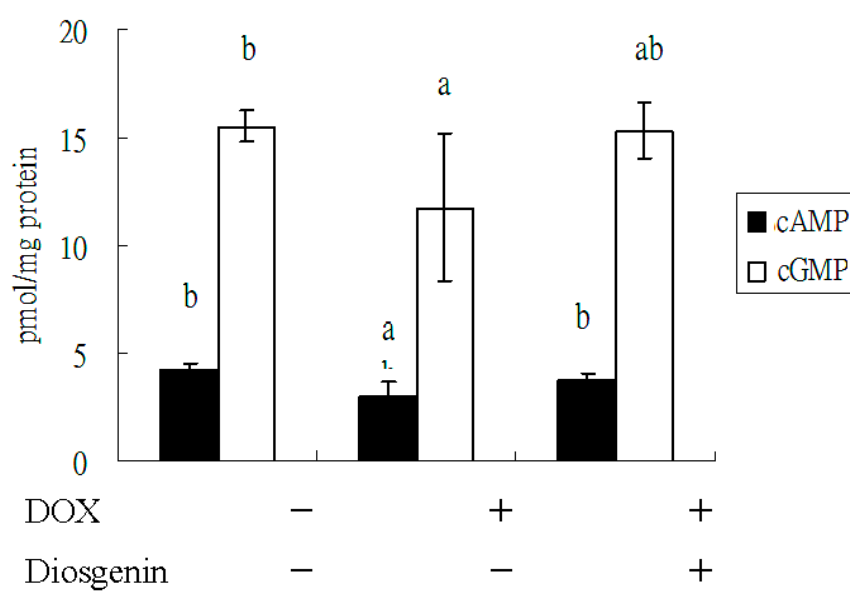

(A)

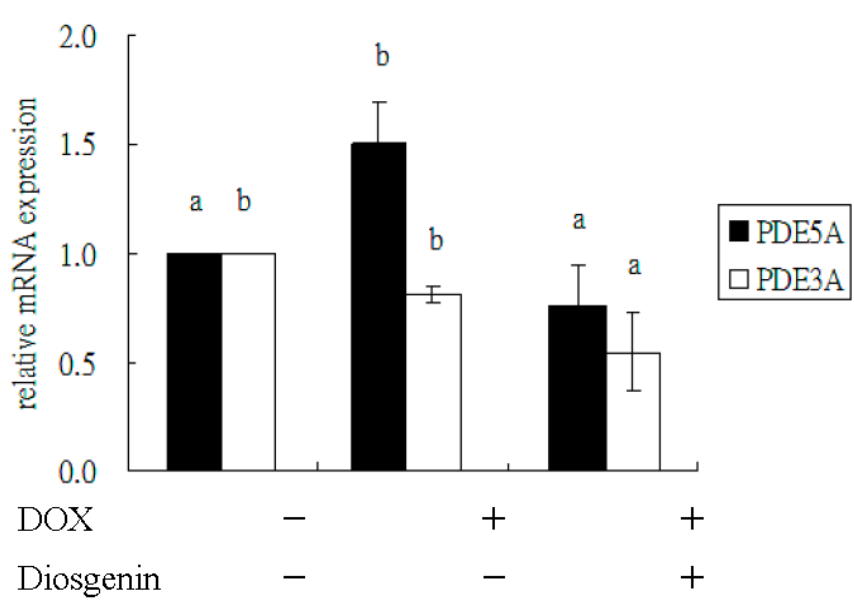

(B)

Figure 1. Effect of diosgenin on levels of cAMP and cGMP (A), and mRNA expressions of PDE5A and PDE3A (B) in heart tissues of mice treated with DOX for four weeks. Values are mean $\pm \mathrm{SD}, n=10$. ${ }^{\mathrm{a}, \mathrm{b}}$ Means in a row without a common letter differ, $p<0.05$.

\subsection{Diosgenin Weakened DOX-Induced Caspase-3 Activation and NF- $\kappa B$ Expression}

To investigate whether the protective effect of diosgenin against DOX occurred because it inhibited apoptotic pathways, we further studied the change in the expression of caspase-3, one marker of apoptosis, in the heart tissue (Figure 2A). Caspase-3 is a cytosolic protein that exists normally as an inactive precursor with a higher molecular weight (about $32 \mathrm{kDa}$ ). It is cleaved proteolytically into low molecular weights $(11,17$, and $20 \mathrm{kDa})$ when a cell undergoes apoptosis [31]. In this study, treatment with DOX induced significantly an increase in cleavage of caspase-3 to 1.53-fold of that of control. In the diosgenin co-treated group, the active fragment was decreased (Figure 2A). As shown in Figure 2B (lane 2), DOX increased significantly the cytosolic concentration of cytochrome c, with a concomitant decrease in the mitochondria, implicating mitochondrial dysfunction due to DOX toxicity [32]. Quantitative data showed that the cytosolic concentration of cytochrome c was increased to 1.87-fold of that of control in the heart tissues of the DOX-treated mice. This effect was significantly suppressed in the DOX plus diosgenin group (lane 3, Figure 2B). The DOX-induced release of cytochrome c from mitochondria was inhibited by $81.6 \%$ in the DOX plus diosgenin group. In addition, mRNA expressions of NF- $\mathrm{BB}$ and transforming growth factor-beta (TGF- $\beta$ ), two factors that are related to inflammation, 
were measured by real-time PCR. DOX treatment augmented the cardiac mRNA expression of NF- $\mathrm{B}$, but not TGF- $\beta$. However, when mice were co-treated with diosgenin, concomitant decreases in their expressions were observed, as compared to the DOX treated group $(p<0.05)$.

\subsection{Diosgenin Restored DOX-Regulated PKA and p38 Activation}

Previous studies found that cAMP-protein kinase A (PKA) pathway promoted cardiomyocyte survival [33]. It has also been shown that p38 mitogen-activated protein kinases (MAPK) pathway is involved in the DOX-induced cardiac oxidative, inflammatory and apoptotic reactions [34]. Consequently, we examined the phosphorylation of PKA and p38, and their total protein levels by Western blotting. As shown in Figure 3, the expressions of phospho-PKA, PKA (Figure 3A), phospho-p38, and p38 (Figure 3B) showed significant increases of about 1.62-, 1.33-, 1.84-, and 1.08-folds, respectively, in the DOX treated group, whereas diosgenin treatment modulated the activation of both protein kinases that were elevated in the presence of DOX (Figure 3).
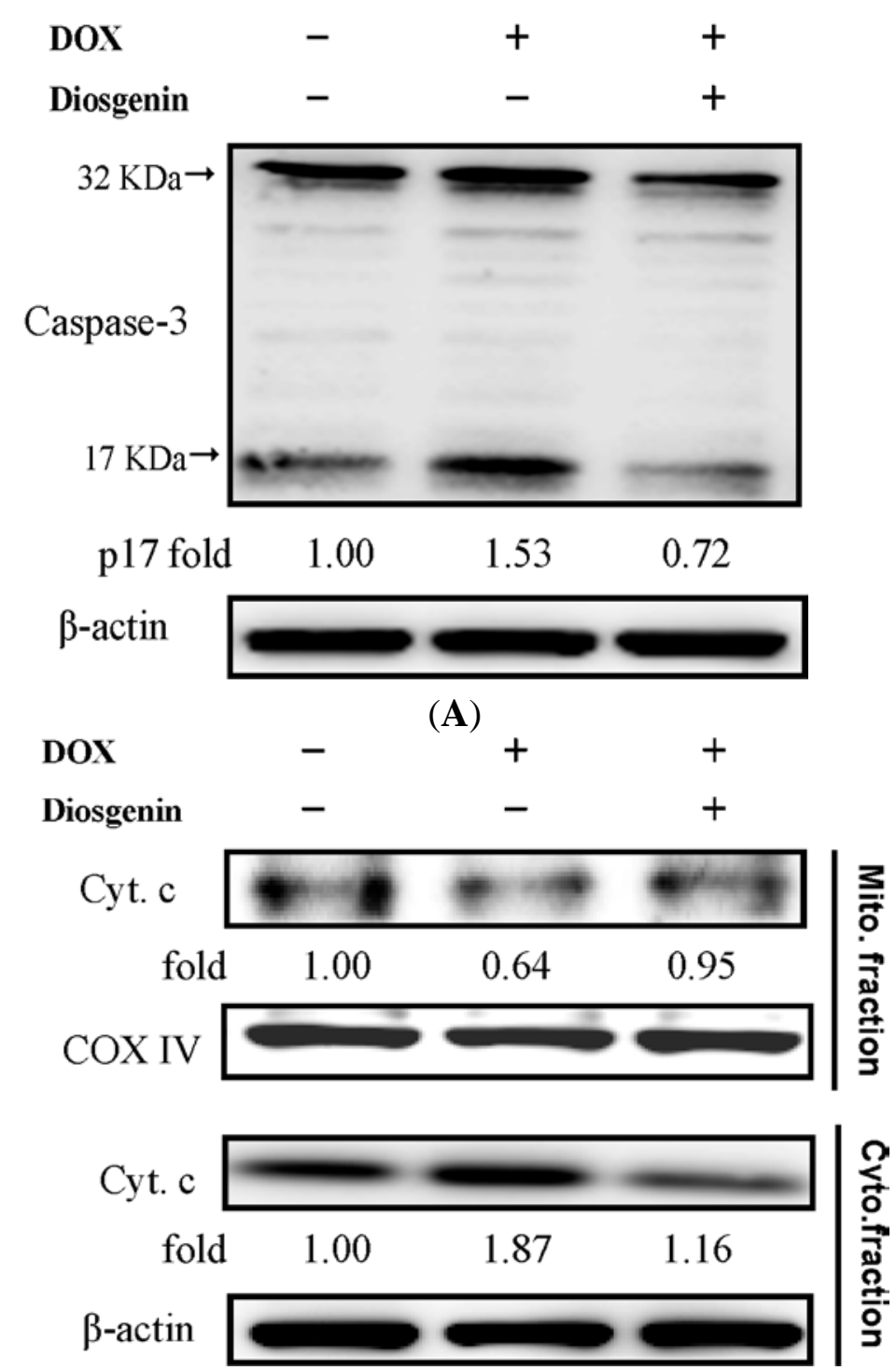

(B)

Figure 2. Cont. 


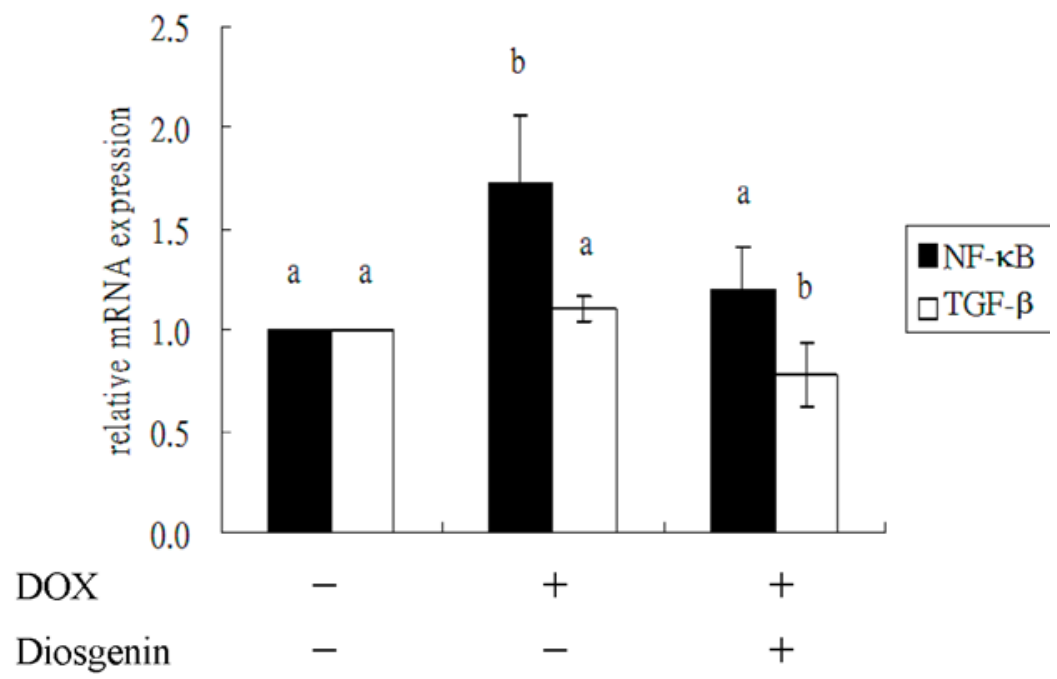

(C)

Figure 2. Effect of diosgenin on the activation of caspase-3 (A); the release of cytochrome c (B); and mRNA expressions of NF- $\kappa$ B and TGF- $\beta$ (C) in heart tissues of mice treated with DOX for four weeks. Values are mean $\pm \mathrm{SD}, n=10{ }^{\mathrm{a}, \mathrm{b}}$ Means in a row without a common letter differ, $p<0.05$. The protein expressions of cytochrome c (cyto. c) from mitochondrial (Mito., line 1-2) and cytosolic (Cyto., line 3-4) fractions. COX IV and $\beta$-actin, respectively, served as an internal control of Mito. and Cyto. fractions. The protein levels above the figures represent relative density of the bands normalized to COX IV (upper panel) or $\beta$-actin (bottom panel). Determined expression of the protein was subsequently quantified by densitometric analysis with that of control being 1.00 -fold, as shown just below the gel data. Results are representative of at least three independent experiments.

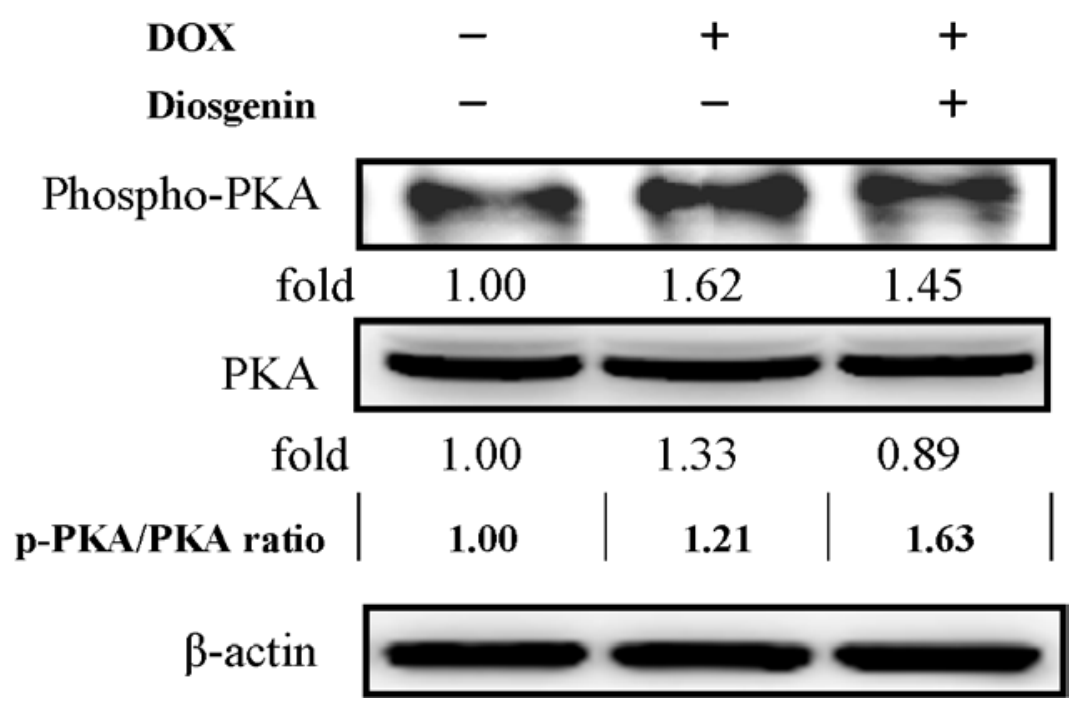

(A)

Figure 3. Cont. 


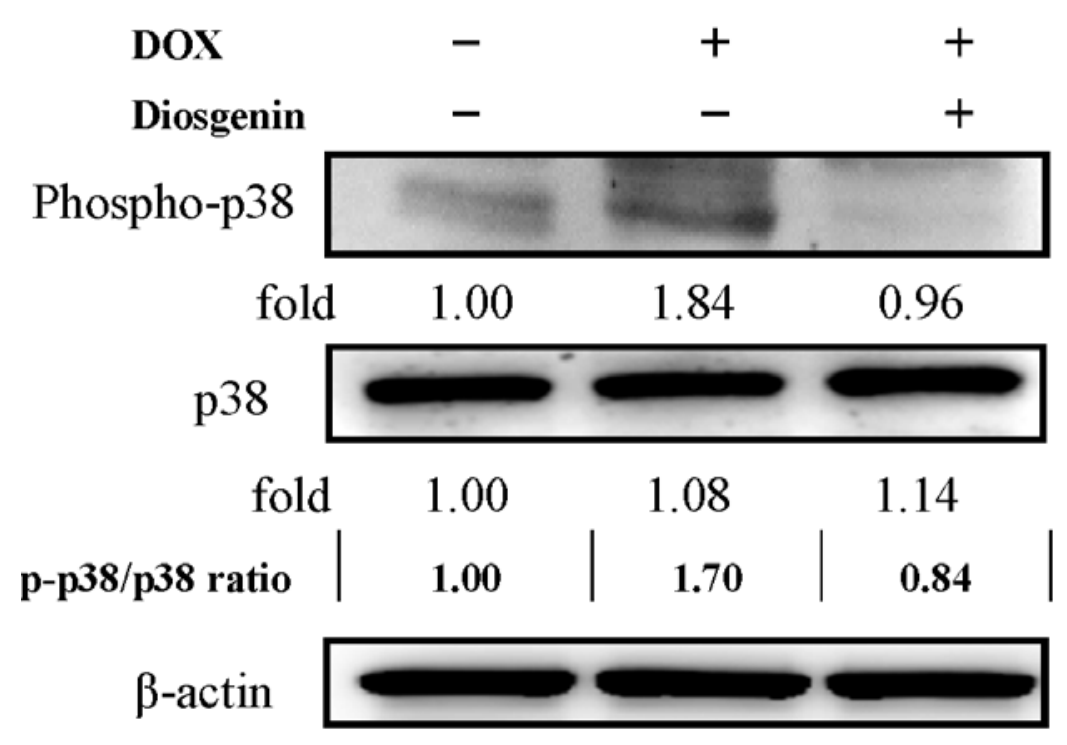

(B)

Figure 3. Effect of diosgenin on the activation of PKA (A) and p38 (B) in heart tissues of mice treated with DOX for four weeks. $\beta$-actin was served as an internal control of protein level. Determined expression of the protein was subsequently quantified by densitometric analysis with that of control being 1.00-fold, as shown just below the gel data. Results are representative of at least three independent experiments. The ratio between phosphorylated and total forms was indicated.

\section{Discussion}

In cancer therapy, especially chemotherapy, DOX has made substantial help in cancer treatment. However, many side effects limit its benefits. Cardiotoxicity, a major side effect of DOX, can be observed in clinical patients and animal studies. The present study examined the possible protective effect of diosgenin, one bioactive compound in yam, on cardiac function in a mouse model of DOX-induced cardiomyopathy. Our data indicated that co-treatment of diosgenin with DOX for four weeks improved cardiac function during the DOX-induced cardiomyopathy, as demonstrated by improvements in body weight, heart weight, as well as in functional parameters including heart rate, blood pressure, and serum levels of LDH, CPK and CK-MB (Table 2). The preservation of heart function was associated with a decrease in the level of oxidative stress (Table 3) and apoptosis (Figure 2A,B) in cardiomyocytes as well as a significant decrease in inflammation status (Figure 2C). In animal models, a similar response has been reported [9,26].

DOX is well known for its cardiac toxicity during chemotherapy for cancer patients. The DOX-induced heart failure has been characterized by the generation of free radicals in the heart tissue [35,36]. In Table 3, the results showed that the activity of SOD was significantly decreased in the DOX-treated animals and the co-treatment of diosgenin reversed the SOD activity with a concomitant rise in the activity of GPx. GSH level was also lowered significantly in the DOX-treated animals, while diosgenin treatment showed a significant increase in GSH level (Table 3). The observed decrease in the activities of SOD and GPx in the DOX-treated animals supports the hypothesis that their decrease are possibly due to excessive use to overcome ROS production. These findings indicate the promising role of 
diosgenin as a cardioprotective agent against the DOX-induced cardiotoxicity. The antioxidant supplements have been suggested to patients with cancer to enhance the benefits of treatment. Antioxidants may also reduce certain types of toxicity associated with chemotherapy [37]. Diosgenin is a potential antioxidant molecule that has a helpful effect on the heart against DOX.

Currently, combinations of anticancer drugs with new agents are being investigated to explore a significant prognostic benefit and improve clinical response. To date, diosgenin has been reported to be very effective against arthritis, gastrointestinal disorders, cardiovascular dysfunction, inflammation and cancer $[17,23,38-40]$. The present study established the cardioprotective effects of diosgenin in the model of DOX-induced mice. The combinations of diosgenin with DOX indeed improved the DOX-induced cardiac toxicity. Further works are needed to clarify whether diosgenin might modulate the cancer killing effect of DOX in vivo. Diosgenin has been shown to exert anti-cancer effects against a wide variety of tumor cells, including colorectal cancer [41], leukemia [42], breast cancer [43], and liver cancer [44]. Moreover, diosgenin potentiated the apoptotic effects of DOX and paclitaxel in human hepatocellular carcinoma HUH-7 cells [44]. Sun et al., indicated that dioscin, the glycoside form of diosgenin, is a potent multidrug resistance reversal agent in the multidrug resistant cell line human hepatocellular carcinoma (HepG2)/DOX, and may be a potential adjunctive agent for tumor chemotherapy [45]. This suggests diosgenin is effective in modulating the anti-cancer effect of DOX in vivo. Future experiments will be carried out to test this possibility and the detail mechanism.

A major adverse side effect associated with DOX use in the clinic is the occurrence of cardiomyopathy and heart failure. Therefore, several reports suggest that the DOX-induced apoptosis plays an important role in cardiotoxicity that is linked to the formation of ROS [46]. ROS production or oxidative stress promotes apoptosis, necrosis and autophagy in cardiomyocytes [47]. Caspase-3 activity can also be influenced by DOX [11]. Antioxidant enzymes form the first line of defense against cardiac tissue damage, and an increased oxidative stress may be due to depletion of antioxidants as reported earlier [36]. In the present study, the DOX-treated group showed an increase in active caspase-3 expression, indicating enhance effect of caspase-3 activity in apoptosis (Figure 2A). After treatment with diosgenin, we observed a significant decrease in the cleavage of caspase-3 (Figure 2A), which is consistent with previous investigations [7,9].

To understand the mechanisms of the diosgenin-mediated protection in the DOX-induced deterioration of cardiac function, we assessed the level of cGMP and cAMP in the heart tissue (Figure 1A). The results of our study showed that diosgenin restored the cGMP and cAMP levels (Figure 1A) as well as decreased the PDE5A expression (Figure 1B) in the heart. cGMP and cAMP are intracellular secondary messengers that mediate multiple cellular functions and morphological processes in the heart, including cardiac protection [48] against apoptosis and hypertrophy [49,50]. At physiological conditions, cGMP and cAMP are inactivated through hydrolysis degradation via PDE. The PDEs vary in their substrate specificity for cGMP and cAMP, among which PDE5 is specific for cGMP, and PDE3 has a mixed specificity for both cAMP and cGMP [51]. In a murine hypertension model, oral supplement of a PDE5A inhibitor, sildenafil, prevents and reverses cardiac hypertrophy, which is mediated by an activation of cGMP-dependent protein kinase [52]. These results suggest that treatment with PDE5 inhibitors might become a promising therapeutic intervention for preventing the DOX-induced cardiotoxicity, which is consistent with some previous studies [53,54]. cAMP promoted cardiomyocyte survival via an effect mediated through the cAMP pathway and the extracellular 
signal-regulated kinase activation [55]. Our results demonstrated that diosgenin can rescue the DOX-induced cardiac cell death effect leading to a down-regulation of cardiomyocyte contractility via cAMP-PKA pathway (Figures 1A and 3A).

Many previous studies revealed that MAPKs play a crucial role in the development of hypertrophy processes such as inflammation and fibrosis, and p38 MAPK can act as a therapeutic target $[34,56]$. It has also been reported that DOX can promote cardiac oxidative, inflammatory and apoptotic reactions via activation of both NF- $\kappa$ B and MAPK pathways in the heart $[57,58]$. Our present study showed that diosgenin declined the mRNA level of NF- $\mathrm{B}$ (Figure 2C) and down-regulated the expression of p-p38 (Figure 3B). Pharmacological inhibition of p38 MAPK protects cardiac myocytes from apoptosis during simulated I/R in vitro, indicating that p38 MAPK functions as a pro-apoptotic signaling effector [59]. It is therefore possible that the inhibitory effect of diosgenin on the DOX-induced cardiotoxicity was conducted via inactivating p38 that subsequently led to a reduction in the apoptotic signaling. However, their relevance needs to be demonstrated.

\section{Conclusions}

DOX-induced cardiomyopathy is an important public health concern because this may limit its therapeutic use for clinical patients. The search for cardioprotective agents will continue to rely on increasing our understanding of the mechanisms of the DOX-induced cardiotoxicity and how to counteract and overcome it. Our results suggest that diosgenin could be a promising agent to prevent the DOX-induced cardiomyopathy via its antioxidant, cGMP pathway, anti-apoptosis and anti-inflammation effects.

\section{Supplementary Materials}

Supplemental Figure S1-Effect of diosgenin on TBARS (A) and GSH (B) levels in kidney tissues of mice treated with DOX for 4 weeks.

\section{Acknowledgments}

This study was supported by the grant from the National Science Council (NSC95-2313-B040-007), Taiwan.

\section{Author Contributions}

Designed overall research experiments: H.-H.L. and J.-H.C. Performed the experiments and data analysis: C.-T.C.; Z.-H.W. and C.-C.H. Collecting and analyzed references: C.-T.C. and Z.-H.W. Wrote the manuscript: H.-H.L. and J.-H.C. All authors read and approved the manuscript.

\section{Conflicts of Interest}

The authors declare no conflict of interest. 


\section{References}

1. Weiss, R.B. The anthracyclines: Will we ever find a better doxorubicin? Semin. Oncol. 1992, 19, 670-686.

2. Jain, D. Cardiotoxicity of doxorubicin and other anthracycline derivatives. J. Nucl. Cardiol. 2000, 7, 53-62.

3. Fu, L.X.; Waagstein, F.; Hjalmarson, A. A new insight into adriamycin-induced cardiotoxicity. Int. J. Cardiol. 1990, 29, 15-20.

4. Singal, P.K.; Iliskovic, N. Adriamycin cardiomyopathy. N. Engl. J. Med. 1998, 339, 900-905.

5. Kalyanaraman, B.; Joseph, J.; Kalivendi, S.; Wang, S.; Konorev, U.; Kotamraju, S. Doxorubicin-induced apoptosis: Implications in cardiotoxicity. Mol. Cell. Biochem. 2002, 234-235, 119-124.

6. Tong, J.; Ganguly, P.K.; Singal, P.K. Myocardial adrenergic changes at two stages of heart failure due to adriamycin treatment in rats. Am. J. Physiol. 1991, 260, H909-H916.

7. Kumar, D.; Kirshenbaum, L.A.; Li, T.; Danelisen, I.; Singal, P.K. Apoptosis in Adriamycin cardiomyopathy and its modulation by probucol. Antioxid. Redox Signal. 2001, 3, 135-145.

8. Hadi, N.; Yousif, N.G.; Al-amran, F.G.; Huntei, N.K.; Mohammad, B.I.; Ali, S.J. Vitamin E and telmisartan attenuates doxorubicin induced cardiac injury in rat through down regulation of inflammatory response. BMC Cardiovasc. Disord. 2012, 12, 63.

9. Lin, M.C.; Yin, M.C. Preventive effects of ellagic acid against doxorubicin-induced cardio-toxicity in mice. Cardiovasc. Toxicol. 2013, 13, 185-193.

10. Nitobe, J.; Yamaguchi, S.; Okuyama, M.; Nozaki, N.; Sata, M.; Miyamoto, T.; Takeishi, Y.; Kubota, I.; Tomoike, H. Reactive oxygen species regulate FLICE inhibitory protein (FLIP) and susceptibility to Fas mediated apoptosis in cardiac myocytes. Cardiovasc. Res. 2003, 57, 119-128.

11. Ueno, M.; Kakinuma, Y.; Yuhki, K.; Murakoshi, N.; Iemitsu, M.; Miyauchi, T.; Yamaguchi, I. Doxorubicin induces apoptosis by activation of caspase-3 in cultured cardiomyocytes in vitro and rat cardiac ventricles in vivo. J. Pharmacol. Sci. 2006, 101, 151-158.

12. Salloum, F.N.; Abbate, A.; Das, A.; Houser, J.E.; Mudrick, C.A.; Qureshi, I.; Hoke, N.N.; Roy, S.K.; Brown, W.R.; Prabhakar, S.; et al. Sildenafil (viagra) attenuates ischemic cardiomyopathy and improves left ventricular function in mice. Am. J. Physiol. Heart Circ. Physiol. 2008, 294, H1398-H1406.

13. Chau, V.Q.; Salloum, F.N.; Hoke, N.N.; Abbate, A.; Kukreja, R.C. Mitigation of the progression of heart failure with sildenafil involves inhibition of RhoA/Rho-kinase pathway. Am. J. Physiol. Heart Circ. Physiol. 2011, 300, H2272-H2279.

14. Das, A.; Xi, L.; Kukreja, R.C. Protein kinase G-dependent cardioprotective mechanism of phosphodiesterase-5 inhibition involves phosphorylation of ERK and GSK3. Biol. Chem. 2008, 283, 29572-29585.

15. Tsai, E.J.; Kass, D.A. Cyclic GMP signaling in cardiovascular pathophysiology and therapeutics. Pharmacol. Ther. 2009, 122, 216-238.

16. Marker, R.E.; Turner, D.L.; Ulshafer, P.R. Sterols. CIV. Diosgenin from certain American plants. J. Am. Chem. Soc. 1940, 62, 2542-2543. 
17. Yamada, T.; Hoshino, M.; Hayakawa, T.; Ohhara, H.; Yamada, H.; Nakazawa, T.; Inagaki, T.; Iida, M.; Ogasawara, T.; Uchida, A.; et al. Dietary diosgenin attenuates subacute intestinal inflammation associated with in domethacin in rats. Am. J. Physiol. 1997, 273, G355-G364.

18. McAnuff, M.A.; Omoruyi, F.O.; Morrison, E.Y.; Asemota, H.N. Plasma and liver lipid distributions in streptozotocin-induced diabetic rats fed sapogenin extract of the Jamaican bitter yam (Dioscorea polygonoides). Nutr. Res. 2002, 22, 1427-1434.

19. McAnuff, M.A.; Harding, W.W.; Omoruyi, F.O.; Jacobs, H.; Morrison, E.Y.; Asemota, H.N. Hypoglycemic effects of steroidal sapogenins isolated from Jamaican bitter yam, Dioscorea polygonoides. Food Chem. Toxicol. 2005, 43, 1667-1672.

20. Son, I.S.; Kim, J.H.; Sohn, H.Y.; Son, K.H.; Kim, J.S.; Kwon, C.S. Antioxidative and hypolipidemic effects of diosgenin, a steroidal saponin of yam (Dioscorea spp.) om high-cholesterol fed rats. Biosci. Biotechnol. Biochem. 2007, 71, 3063-3071.

21. Hsu, C.C.; Huang, Y.C.; Yin, M.C.; Lin, S.J. Effect of yam (Dioscorea alata vs. Dioscorea japonica) on gastrointestinal function and antioxidant activity in mice. J. Food Sci. 2006, 71, 513-516.

22. Vasanthi, H.R.; Mukherjee, S.; Ray, D.; Pandian Jayachandran, K.S.; Lekli, I.; Das, D.K. Protective role of air potato (Dioscorea bulbifera) of yam family in myocardial ischemic reperfusion injury. Food Funct. 2010, 1, 278-283.

23. Jayachandran, K.S.; Vasanthi, H.R.; Rajamanickam, G.V. Antilipoperoxidative and membrane stabilizing effect of diosgenin, in experimentally induced myocardial infarction. Mol. Cell. Biochem. 2009, 327, 203-210.

24. Choi, K.W.; Park, H.J.; Jung, D.H.; Kim, T.W.; Park, Y.M.; Kim, B.O.; Sohn, E.H.; Moon, E.Y.; Um, S.H.; Rhee, D.K.; et al. Inhibition of TNF- $\alpha$-induced adhesion molecule expression by diosgenin in mouse vascular smooth muscle cells via downregulation of the MAPK, Akt and NF-kB signaling pathways. Vasc. Pharmacol. 2010, 53, 273-280.

25. Pari, L.; Monisha, P.; Mohamed Jalaludeen, A. Beneficial role of diosgenin on oxidative stress in aorta of streptozotocin induced diabetic rats. Eur. J. Pharmacol. 2012, 691, 143-150.

26. Patil, L.; Balaramanb, R. Effect of green tea extract on doxorubicin induced cardiovascular abnormalities: Antioxidant action. Iran. J. Pharm. Res. 2011, 10, 89-96.

27. Chen, J.H.; Tsai, C.W.; Wang, C.P.; Lin, H.H. Anti-atherosclerotic potential of gossypetin via inhibiting LDL oxidation and foam cell formation. Toxicol. Appl. Pharmacol. 2013, 272, 313-324.

28. Chung, H.; Jung, S.H.; Ryu, J.K.; Kim, B.; Kim, H.S.; Yang, S.K. Isolation and characterization of smooth muscle cells from rat corpus cavernosum tissue for the study of erectile dysfunction. Korean J. Urol. 2012, 53, 556-563.

29. Zhang, C.; Feng, Y.; Qu, S.; Wei, X.; Zhu, H.; Luo, Q.; Liu, M.; Chen, G.; Xiao, X. Resveratrol attenuates doxorubicin-induced cardiomyocyte apoptosis in mice through SIRT1-mediated deacetylation of p53. Cardiovasc. Res. 2011, 90, 538-545.

30. Elberry, A.A.; Abdel-Naim, A.B.; Abdel-Sattar, E.A.; Nagy, A.A.; Mosli, H.A.; Mohamadin, A.M.; Ashour, O.M. Cranberry (Vaccinium macrocarpon) protects against doxorubicin-induced cardiotoxicity in rats. Food Chem. Toxicol. 2010, 48, 1178-1184. 
31. Susin, S.A.; Zamzami, N.; Castedo, M.; Daugas, E.; Wang, H.G.; Geley, S.; Fassy, F.; Reed, J.C.; Kroemer, G. The central executioner of apoptosis: Multiple connections between protease activation and mitochondria in Fas/APO-1/CD95- and ceramide-induced apoptosis. J. Exp. Med. 1997, 186, 25-37.

32. Green, P.S.; Leeuwenburgh, C. Mitochondrial dysfunction is an early indicator of doxorubicin-induced apoptosis. Biochim. Biophys. Acta 2002, 1588, 94-101.

33. Maurice, D.H.; Palmer, D.; Tilley, D.G.; Dunkerley, H.A.; Netherton, S.J.; Raymond, D.R.; Elbatarny, H.S.; Jimmo, S.L. Cyclic nucleotide phosphodiesterase activity, expression, and targeting in cells of the cardiovascular system. Mol. Pharmacol. 2003, 64, 533-546.

34. Dhalla, N.S.; Mueller, A.L. Protein kinases as drug development targets for heart disease therapy. Pharmaceuticals 2010, 3, 2111-2145.

35. Naidu, M.U.R.; Vijay Kumar, K.; Krishna Mohan, I.; Sundaram, C.; Singh, S. Protective effect of Gingko biloba extract against doxorubicin-induced cardiotoxicity in mice. Indian J. Exp. Biol. 2002, 40, 894-900.

36. Hardina, R.; Gersl, V.; Klimtova, I.; Simunek, T.; Machackova, J.; Adamcova, M. Anthracycline induced cardiotoxicity. Acta Medica. 2000, 43, 75-82.

37. Ladas, E.J.; Jacobson, J.S.; Kennedy, D.D.; Teel, K.; Fleischauer, A.; Kelly, K.M. Antioxidants and cancer therapy: A systematic review. J. Clin. Oncol. 2004, 22, 517-528.

38. Liagre, B.; Vergne-Salle, P.; Corbiere, C.; Charissoux, J.L.; Beneytout, J.L. Diosgenin, a plant steroid, induces apoptosis in human rheumatoid arthritis synoviocytes with cyclooxygenase-2 overexpression. Arthritis Res. Ther. 2004, 6, R373-R383.

39. Trouillas, P.; Corbière, C.; Liagre, B.; Duroux, J.L.; Beneytout, J.L. Structurefunction relationship for saponin effects on cell cycle arrest and apoptosis in the human osteosarcoma cells: A molecular modelling approach of natural molecules structurally close to Diosgenin. Bioorg. Med. Chem. 2005, 13, 1141-1149.

40. Manivannan, J.; Shanthakumar, J.; Arunagiri, P.; Raja, B.; Balamurugan, E. Diosgenin interferes coronary vasoconstriction and inhibits osteochondrogenic transdifferentiation of aortic VSMC in CRF rats. Biochimie 2014, 102, 183-187.

41. Wang, S.L.; Cai, B.; Cui, C.B.; Liu, H.W.; Wu, C.F.; Yao, X.S. Diosgenin-3-OalphaL-rhamnopyranosyl-(14)-beta-D-glucopyranoside obtained as a new anticancer agent from Dioscorea futschauensis induces apoptosis on human colon carcinoma HCT-15 cells via mitochondria-controlled apoptotic pathway. J. Asian Nat. Prod. Res. 2004, 6, 115-125.

42. Liu, M.J.; Wang, Z.; Ju, Y.; Wong, R.N.; Wu, Q.Y. Diosgenin induces cell cycle arrest and apoptosis in human leukemia K562 cells with the disruption of $\mathrm{Ca}^{2+}$ homeostasis. Cancer Chemother. Pharmacol. 2005, 55, 79-90.

43. Srinivasan, S.; Koduru, S.; Kumar, R.; Venguswamy, G.; Kyprianou, N.; Damodaran, C. Diosgenin targets Akt-mediated prosurvival signaling in human breast cancer cells. Int. J. Cancer 2009, 125, 961-967.

44. Li, F.; Fernandez, P.P.; Rajendran, P.; Hui, K.M.; Sethi, G. Diosgenin, a steroidal saponin, inhibits STAT3 signaling pathway leading to suppression of proliferation and chemosensitization of human hepatocellular carcinoma cells. Cancer Lett. 2010, 292, 197-207. 
45. Sun, B.T.; Zheng, L.H.; Bao, Y.L.; Yu, C.L.; Wu, Y.; Meng, X.Y.; Li, Y.X. Reversal effect of Dioscin on multidrug resistance in human hepatoma HepG2/adriamycin cells. Eur. J. Pharmacol. 2011, 654, 129-134.

46. Menna, P.; Salvatorelli, E.; Cairo, G.; Gianni, L. Anthracyclines: Molecular advances and pharmacologic developments in antitumor activity and cardiotoxicity. Pharmacol. Rev. 2004, 56, 185-229.

47. Zhang, Y.W.; Shi, J.; Li, Y.J.; Wei, L. Cardiomyocyte death in doxorubicin-induced cardiotoxicity. Arch. Immunol. Ther. Exp. (Warsz) 2009, 57, 435-445.

48. Das, A.; Smolenski, A.; Lohmann, S.M.; Kukreja, R.C. Cyclic GMP-dependent protein kinase I alpha attenuates necrosis and apoptosis following ischemia/reoxygenation in adult cardiomyocyte. J. Biol. Chem. 2006, 281, 38644-38652.

49. Tomita, H.; Nazmy, M.; Kajimoto, K.; Yehia, G.; Molina, C.A.; Sadoshima, J. Inducible cAMP early repressor (ICER) is a negative-feedback regulator of cardiac hypertrophy and an important mediator of cardiac myocyte apoptosis in response to beta-adrenergic receptor stimulation. Circ. Res. 2003, 93, 12-22.

50. Booz, G.W. Putting the brakes on cardiac hypertrophy: Exploiting the NO-cGMP counter-regulatory system. Hypertension 2005, 45, 341-346.

51. Kulkarni, S.K.; Patil, C.S. Phosphodiesterase 5 enzyme and its inhibitors: Update on pharmacological and therapeutical aspects. Methods Find Exp. Clin. Pharmacol. 2004, 26, 789-799.

52. Takimoto, E.; Champion, H.C.; Li, M.; Belardi, D.; Ren, S.; Rodriguez, E.R.; Bedja, D.; Gabrielson, K.L.; Wang, Y.; Kass, D.A. Chronic inhibition of cyclic GMP phosphodiesterase 5A prevents and reverses cardiac hypertrophy. Nat. Med. 2005, 11, 214-222.

53. Westermann, D.; Becher, P.M.; Lindner, D.; Savvatis, K.; Xia, Y.; Fröhlich, M.; Hoffmann, S.; Schultheiss, H.P.; Tschöpe, C. Selective PDE5A inhibition with sildenafil rescues left ventricular dysfunction, inflammatory immune response and cardiac remodeling in angiotensin II-induced heart failure in vivo. Basic Res. Cardiol. 2012, 107, 308.

54. Zeidan, A.; Siam, A.; Al Kaabba, A.; Mohammad, M.; Khatib, S. The ability of phosphodiesterase-5 inhibitors sildenafil and ordonafil to reverse L-NAME induced cardiac hypertrophy in the rabbit: Possible role of calcineurin and p38. Can. J. Physiol. Pharmacol. 2012, 90, 1247-1255.

55. Henaff, M.; Hatem, S.N.; Mercadier, J.J. Low catecholamine concentrations protect adult rat ventricular myocytes against apoptosis through cAMP dependent extracellular signal-regulated kinase activation. Mol. Pharmacol. 2000, 58, 1546-1553.

56. Liao, P.; Georgakopoulos, D.; Kovacs, A.; Zheng, M.; Lerner, D.; Pu, H.; Saffitz, J.; Chien, K.; Xiao, R.P.; Kass, D.A.; et al. The in vivo role of p38 MAP kinases in cardiac remodeling and restrictive cardiomyopathy. Proc. Natl. Acad. Sci. USA 2001, 98, 12283-12288.

57. Trivedi, P.P.; Kushwaha, S.; Tripathi, D.N.; Jena, G.B. Cardioprotective effects of hesperetin against doxorubicin-induced oxidative stress and DNA damage in rat. Cardiovasc. Toxicol. 2011, 11, 215-225.

58. Chen, Y.L.; Loh, S.H.; Chen, J.J.; Tsai, C.S. Urotensin II prevents cardiomyocyte apoptosis induced by doxorubicin via Akt and ERK. Eur. J. Pharmacol. 2012, 680, 88-94. 
59. Yue, T.L.; Wang, C.; Gu, J.L.; Ma, X.L.; Kumar, S.; Lee, J.C.; Feuerstein, G.Z.; Thomas, H.; Maleeff, B.; Ohlstein, E.H. Inhibition of extracellular signal-regulated kinase enhances ischemia/reoxygenation-induced apoptosis in cultured cardiac myocytes and exaggerates reperfusion injury in isolated perfused heart. Circ. Res. 2000, 86, 692-699.

(C) 2015 by the authors; licensee MDPI, Basel, Switzerland. This article is an open access article distributed under the terms and conditions of the Creative Commons Attribution license (http://creativecommons.org/licenses/by/4.0/). 